\title{
GMR
}

\section{Analysis of allopolyploidy-induced rapid genetic and epigenetic changes and their relationship in wheat}

\author{
T. Qiu ${ }^{1,2,3}$, Y.Z. Dong ${ }^{1,3}$, X.M. Yu ${ }^{3}$, N. Zhao ${ }^{4}$ and Y.F. Yang ${ }^{2}$ \\ ${ }^{1}$ School of Life Sciences, Changchun Normal University, Changchun, China \\ ${ }^{2}$ Key Laboratory of Vegetation Ecology, Ministry of Education, \\ Institute of Grassland Science, Northeast Normal University, Changchun, China \\ ${ }^{3}$ Key Laboratory of Molecular Epigenetics, Ministry of Education, \\ Northeast Normal University, Changchun, China \\ ${ }^{4}$ Faculty of Agronomy, Jilin Agricultural University, Changchun, China \\ Corresponding author: N. Zhao \\ E-mail: zhaonjlau@yahoo.com
}

Genet. Mol. Res. 16 (2): gmr16029303

Received September 19, 2016

Accepted February 17, 2017

Published April 5, 2017

DOI http://dx.doi.org/10.4238/gmr16029303

Copyright (C) 2017 The Authors. This is an open-access article distributed under the terms of the Creative Commons Attribution ShareAlike (CC BY-SA) 4.0 License.

\begin{abstract}
We used the conventional and methylation-sensitive randomly amplified polymorphic DNA (RAPD) and inter-simple sequence repeat (ISSR) analyses to assess genome-wide changes and explore the relationships between genetic and epigenetic variations among individuals of a newly synthesized allohexaploid wheat line whose genomic constitution is identical to that of the natural common wheat, compared with its parent plants and a natural counterpart named Chinese Spring. We found rapid, extensive, and predominantly consistent non-Mendelian changes in the form of genetic and DNA methylation variations in the allohexaploid individuals. Specifically, at least 30-40\% of the epigenetic component was truly independent of genetic changes, which answered a critical question, i.e. its autonomy in relation to the genetic context. Striking correlations were detected between genetic and
\end{abstract}

Genetics and Molecular Research 16 (2): gmr16029303 
epigenetic changes. Interestingly, as previously reported, the paternally donated nuclear genomes showed more genetic changes than the maternally donated ones; the loss of paternal bands was significantly correlated with the hypomethylation of CG or CHG sequences, suggesting an unknown link between genetic instability and hypomethylation. Sequence analysis indicated that most variations occurred in the cellular genes and sequences related to transposable elements. Based on these findings, the possible mechanisms and effects of the genomic changes in allopolyploid speciation and evolution were discussed.

Key words: Synthetic allohexaploid wheat; Genetic variations; Methylation changes; Randomly amplified polymorphic DNA (RAPD); Evolution; Inter-simple sequence repeat (ISSR)

\section{INTRODUCTION}

Allopolyploidization entails the combination of two or more divergent genomes from different species into a common nucleus and one parent's cytoplasm, constituting a radical and rapid mode of speciation (Feldman et al., 2012; Madlung and Wendel, 2013; Li et al., 2015). Allopolyploidy has long been recognized as a powerful evolutionary process in higher plants, having produced many wild and cultivated species (Liu and Wendel, 2002; Hegarty and Hiscock, 2008; Ainouche and Jenczewski, 2010). However, the mechanisms by which an allopolyploid is created in the short term and how it confers selective advantages on a longer timescale are poorly understood. Recent studies in several model plants have addressed this issue and revealed that the newly formed allopolyploid genomes are extremely dynamic and often associated with rapid and extensive genomic changes in multiple layers, including genetic/epigenetic, transcriptomic, and proteomic changes, which transgress Mendelian principles (Song et al., 1995; Liu et al., 1998, 2009; Zhao et al., 2011a). Such changes would help the plants to overcome a 'bottleneck' of sterility and instability and generate a vast reservoir of variation for evolutionary success. However, it should be noted that the genomic changes are not an automatic response generated by all newly synthesized allopolyploid plants. In Brassica, Spartina anglica, and Gossypium, no extensive genetic changes are associated with polyploidization (Liu and Wendel, 2002), suggesting that this phenomenon of polyploidization-induced genetic changes is a species-dependent trait.

The polyploid wheat provides a classic example of evolution and domestication through allopolyploidy. In particular, the hexaploid common wheat Triticum aestivum L. (2n $=6 \mathrm{x}=42)$ is the most important and widely grown food crop in the world, and its creation involved two allopolyploidization events (interspecific hybridization before or followed by genome doubling). The interspecific hybridization event, which occurred approximately $0.4-$ 0.5 mya, involved the formation of tetraploid wheat, T. turgidum ssp dicoccoides, through allotetraploidization between the A-genome-containing diploid wheat species, T. uratu, and an S-genome- (or a closely related genome)-containing diploid goatgrass species most likely akin to Aegilops speltoides. Subsequently, the tetraploid wheat T. turgidum ssp dicoccoides hybridized again with the D-genome-containing diploid goatgrass species A.tauschii to form the hexaploid wheat species T. aestivum (Feldman, 2001). Some recent studies suggest that an extensive and heritable epigenetic remodeling coupled with a preponderance of genetic

Genetics and Molecular Research 16 (2): gmr16029303 
stability is generally associated with nascent allohexaploidization in wheat (Zhao et al., 2011b). This conclusion is at odds with the previous studies conducted on the newly synthesized tetraand hexapolyploidization wheat (Ozkan et al., 2001; Shaked et al., 2001; Dong et al., 2005; Kraitshtein et al., 2010; Qi et al., 2010). These findings imply that the phenomena of genetic changes associated with allopolyploidization might be the genotype-dependent traits in wheat. A common character of these synthesized (allotetraploid/allohexaploid) wheat lines is extensive epigenetic variation, suggesting that it might play a key role in polyploid formation and evolution (Rapp and Wendel, 2005). Though the genetic and epigenetic variations could be simultaneously induced in the process of polyploidization, a few attempts have been made to explore the possible intrinsic relationships between these two types of variations. To our knowledge, the relationship between these variations has been explored in tetraploid wheat (Qi et al., 2010).

Several DNA markers have been successfully used in previous studies to characterize the genomic stability of allopolyploids. Among these markers, randomly amplified polymorphic DNA (RAPD) and inter-simple sequence repeat (ISSR) have been favored because of their sensitivity, simplicity, and cost-effectiveness (Wolfe et al., 1998; Guo et al., 2006). They have also been applied in unbiased and global studies on plants, such as Spartina anglica (Liu and Wendel, 2002). Specifically, the present study aimed to extend previous studies by investigating the generality of allohexaploidization-induced rapid genetic changes in wheat using distinct markers. The limitations of RAPD techniques, such as reputedly low replicability, can be improved by performing reproducibility tests and by using a conservative scoring approach (Kjølner et al., 2004). Importantly, to investigate the correlations between genetic and epigenetic variations, the methylation-sensitive ISSR/RAPDs were developed simultaneously. Using these methods, we found rapid and extensive genetic and DNA methylation alterations in the newly synthesized allohexaploid wheat, most of which were consistent and some epigenetic and genetic variations were correlated. The possible causes and effects in polyploid establishment and evolution are discussed.

\section{MATERIAL AND METHODS}

\section{Plant materials}

Two randomly chosen individuals (designated SY1 and SY2) of a seventh-generation synthetic allohexaploid wheat (genome AABBDD, code no. XX340), its maternal parent plant of wild tetraploid wheat, Triticum turgidum ssp dicoccoides (genome AABB, accession TTD09), and its paternal parent plant of diploid wild goat grass, Aegilops tauschii (genome DD, accession TQ01) were used. These plants were kindly provided by Professor M. Feldman of the Weizmann Institute of Science, Israel. A standard laboratory cultivar of common wheat, Triticum aestivum L. 'Chinese Spring' (CS), was also included for comparison. All plants were grown in the growth chambers maintained at $22 / 18^{\circ} \mathrm{C}$ day/night with a 12 -h day length.

\section{Analysis of conventional and methylation-sensitive RAPD and ISSR}

Genomic DNA was isolated from the young expanded leaves of individual plants in the same developmental phase using the cetyltrimethylammonium bromide (CTAB) method (Kidwell and Osborn, 1992). For consistency and ease of comparison, three forms of genomic 
DNA-non-digested, MspI-digested, and HpaII-digested-from each individual were used as templates for simultaneous amplification with RAPD and ISSR primers. The PCRs and RAPD or ISSR programs were performed as originally described (Ammiraju et al., 2001; Guo et al., 2006). In the methylation-sensitive analysis, the isoschizomers HpaII and MspI have the same recognition sequence of 5'-CCGG-3', but have different sensitivities to the methylation state of cytosine: HpaII cannot cut if either of the cytosines are fully (double-strand) methylated, whereas MspI cannot cut if the external cytosine is methylated (McClelland et al., 1994). Therefore, for a given genotype, a comparison of the amplification products from HpaII and MspI allows for the identification of some types of methylation states. Specifically, the bands present in the HpaII digestion, but absent from the corresponding Ms $\mathrm{I}$ digestion, indicate that the internal cytosine is fully methylated. For clarity, we hereby define this type of cytosine methylation as CG methylation. Bands absent from the HpaII digestion, but present in the corresponding $M s p$ I digestion, indicate that the external cytosine is hemi-methylated. Likewise, we define this type as $\mathrm{CHG}$ methylation. Accordingly, four patterns of methylation changes: CG hypo, CHG hypo, CG hyper, and CHG hyper, could be assessed in the cases when either of the two methylation states mentioned above and other methylation states change between each other. For example, bands present in both HpaII and MspI in the parent(s), but present in only HpaII or MspI in the progeny, indicate CHG hypo; bands absent from both HpaII and $M s p \mathrm{I}$ in the parent(s), but present in MspI or HpaII in the progeny, indicate CHG hyper or CG hyper, respectively; and bands present in MspI or HpaII in the parent(s), but absent from both HpaII and MspI in the progeny, indicate CHG hypo or CG hypo, respectively.

The bands that showed genetic alterations in the conventional analysis and revealed complete additivity at the same sites in the methylation-sensitive analysis were ruled out because of the conformationally constrained templates. Two technical replications were performed starting from independent DNA isolation; only clear and completely reproducible bands were scored. The data were transformed into a binary character matrix: "1" for presence or "0" for absence at a particular position.

\section{Statistical analysis}

Possible correlations between genetic and epigenetic alterations were tested using the Pearson correlation analysis based on the data for variation frequencies. Specifically, the bivariate two-tailed Pearson's correlation analyses were executed within the software SPSS 19 for Windows (http://www.spss.com/statistics/).

\section{Sequencing of variant bands and Southern blot hybridization}

Bands of interest were eluted from the gel, purified, and ligated into the pGEM-T vector (Promega, Madison, WI, USA) and sequenced. Homology searches were conducted using the programs BlastN or BlastX against the GenBank database on the National Centre for Biotechnology Information (NCBI) website.

To validate the methylation alterations revealed by the methylation-sensitive RAPD/ ISSR, we selected six cloned DNA segments representing different types of methylation alterations as probes. Genomic DNA was digested with EcoRI, EcoRI + HpaII, and EcoRI + MspI (New England BioLabs, Inc., Ipswich, MA, USA). To ensure complete digestion, an excess of enzymes (10 units enzyme/ $\mu$ g DNA) was used, and the incubation time was

Genetics and Molecular Research 16 (2): gmr16029303 
extended to $\sim 48 \mathrm{~h}$. Southern hybridizations were performed as previously described (Dong et al., 2005).

\section{RESULTS}

\section{Rapid genetic changes occurred in the synthetic allohexaploid wheat}

The conventional RAPD and ISSR analysis enables an assessment of the allopolyploidyassociated genetic changes in a quantitative and unbiased manner from a genome-wide perspective. Using 16 and 43 selective primers in RAPD and ISSR amplifications, respectively (Table S1), 136 and 258 bands (loci) for the two parental species were scored, of which the majority ( 76.5 and $72.5 \%$ ) were polymorphic bands that were almost evenly distributed in the two parents (Table S2). According to the null hypothesis for genome stability in an interspecies hybrid or allopolyploid genome (Wendel, 2000), if no genetic changes occurred, then the bands should show an additive pattern of both parents, given the dominant nature of the marker and homozygous parents. In contrast to this assumption, deviations were observed for all studied allohexaploid individuals. The changes could be grouped into two categories: loss of a parental band and gain of a novel band. In the RAPD profile, the numbers of lost bands from SY1 and SY2 were 39 and 42, respectively, and two new bands appeared de novo in two synthetic allohexaploid individuals; the frequency of alteration in the natural counterpart (Chinese Spring: CS) was relatively higher, and the numbers of losses and gains were 62 and 9, respectively (Figure 1 and Table S2). In the ISSR patterns, 83-84 bands were absent, and 12-13 novel bands appeared in the synthetic progenies; in the natural counterpart, 105 bands disappeared, and 18 new bands appeared (Figure 1 and Table S2). Overall, 30.7-39.5\% bands in the synthetic allohexaploid wheat showed non-additivity, while $51.23-56.43 \% \mathrm{f}$ bands in the CS showed non-additivity (including two markers). Both RAPD and ISSR analyses revealed that most of the lost bands were from the paternal parent, suggesting a type of directional genome change. The ratio of monomorphic to polymorphic bands was approximately $1 / 3$. Both these findings coincided with the previous report on two synthetic allohexaploid wheat lines (Figure 2 and Table S2) (Nie et al., 2008).

\section{Rapid epigenetic changes occurred in the synthetic allohexaploid wheat}

The methylation-sensitive RAPD/ISSR analysis was executed to conduct a genomewide screening for allopolyploidy-associated epigenetic changes in the form of cytosine methylation at the 5'-CCGG-3' sites, which are the predominant sites for methylation modification in eukaryotes. In the methylation-sensitive analysis using the same RAPD and ISSR primers, 165 and 281 clear and reproducible bands were scored, respectively (Table S3). If no epigenetic changes had occurred in the synthetic allohexaploid wheat line, we would have expected complete additivity. In contrast, four patterns of methylation changes (CG hypo, CHG hypo, CG hyper, and CHG hyper) occurred in all allohexaploid individuals studied (Figure 3 and Table S3). Of the 165 bands scored in the RAPD banding patterns of the parents and their two progenies, 22-23 bands displayed deviation. Regarding CS, 28 bands deviated out of the total 174 bands scored. In the ISSR patterns, of the 279-281 bands scored, a total of 42-44 bands showed deviations, while 53 out of 290 bands deviated in CS (Figures 1 and 3; Table S3). Therefore, in two methylation sensitive analyses, $13.33-15.66 \%$ bands 
from the synthetic allohexaploid wheat showed non-additivity, and $16.09-18.28 \%$ bands from CS showed deviation. Among the four patterns of methylation changes, CHG hypo was the most abundant and CG hyper was the least abundant. In addition, no significant differences in methylation levels for CG and CNG were observed between allohexaploid individuals and their parental species (data not shown).

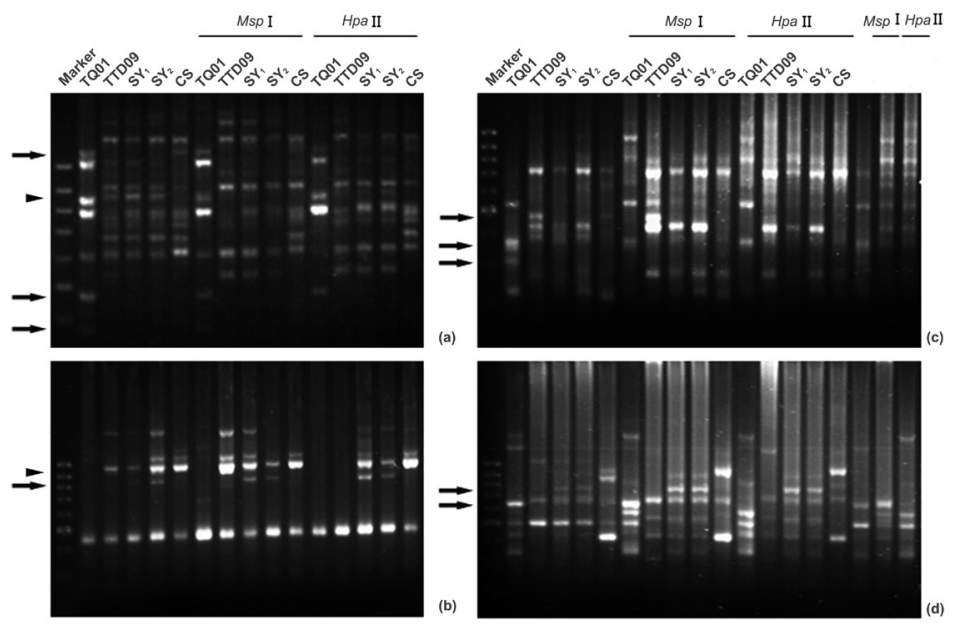

Figure 1. Examples of conventional and methylation-sensitive randomly amplified polymorphic DNA (RAPD) (a) and inter-simple sequence repeat (ISSR) (b, c, d) patterns of two individuals of a synthetic allohexaploid wheat (designated $\mathrm{SY}_{1}$ and $\mathrm{SY}_{2}$ ), its diploid (TQ01) and tetraploid (TTD09) parents, and the natural common wheat, Chinese Spring (CS) obtained using primers A17 (a), W24 (b), W6 (c), and W8 (d). Arrows and arrowheads denote genetic changes, including the loss of a parental band(s) or gain of a novel band(s) and DNA methylation changes, respectively. Lanes 17, 18, and 19 in (c) and (d) represent amplifications with the non-digested, MspI- or HpaIIdigested DNA of the man-made mixed parents to exclude PCR artifacts.

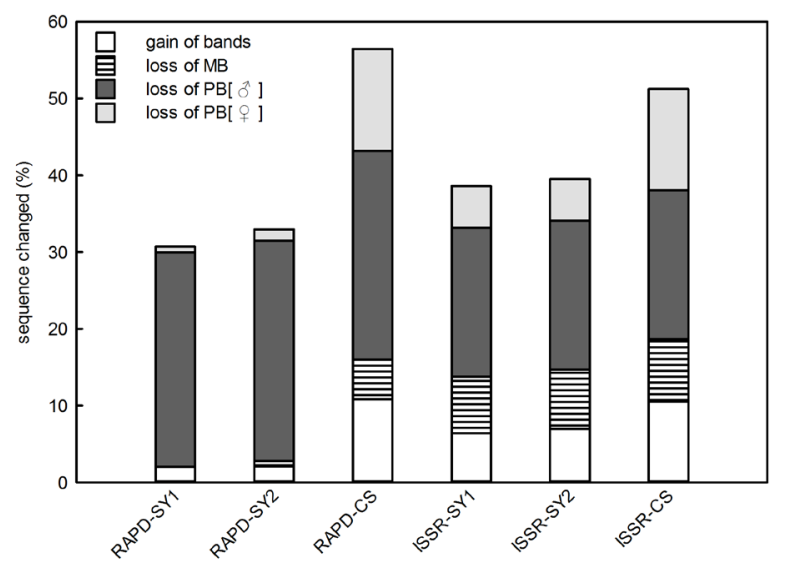

Figure 2. Tabulated genetic changes in each individual of the synthetic and natural allohexaploid wheat as revealed by conventional randomly amplified polymorphic DNA (RAPD) and inter-simple sequence repeat (ISSR). The percentages of the changes involving the loss of parental bands from the maternal parent, the paternal parent, and monomorphic bands along with gain of novel bands.

Genetics and Molecular Research 16 (2): gmr16029303 


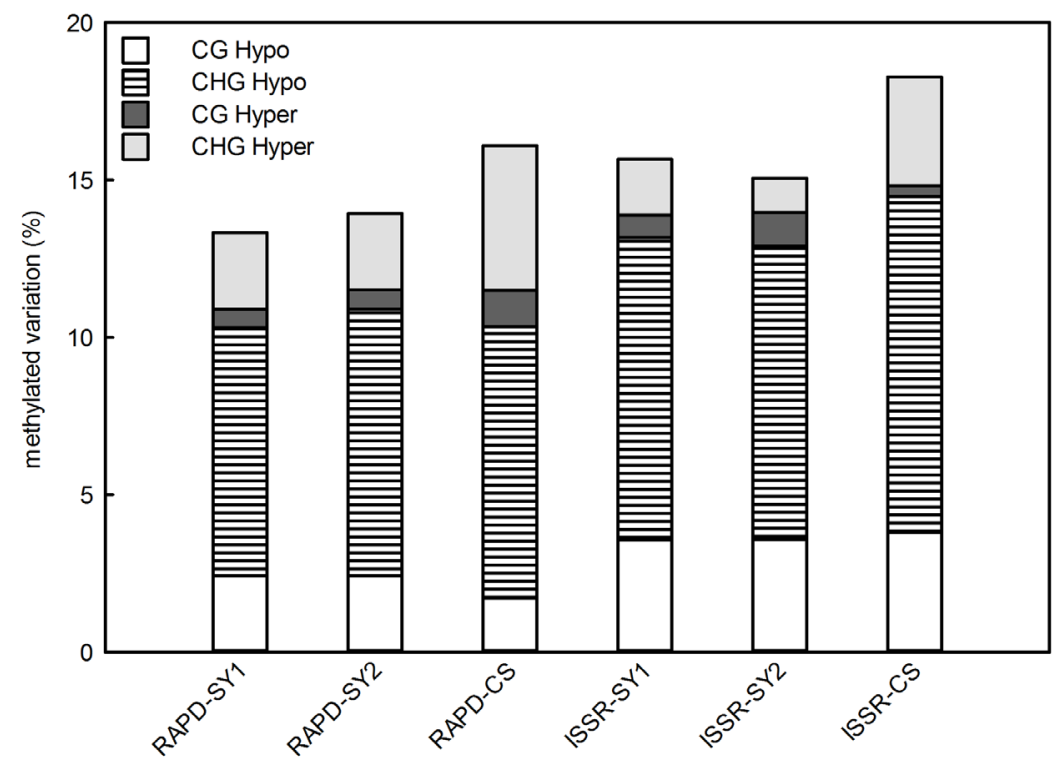

Figure 3. Tabulated epigenetic changes in each individual of the synthetic and natural allohexaploid wheat revealed by methylation-sensitive randomly amplified polymorphic DNA (RAPD) and inter-simple sequence repeat (ISSR). The percentages of the changes involving four DNA methylation patterns (CG hypo, CHG hypo, CG hyper, and CHG hyper) are presented.

\section{Genetic and methylation alterations are largely consistent in allohexaploids and CS}

Both consistent and random changes were identified in the conventional and methylation-sensitive analyses performed on the synthetic allohexaploids. Here, "consistent" refers to the changes that simultaneously occurred in both of the two randomly chosen individuals of the synthetic allohexaploid line; the changes were often conserved in the natural counterpart, CS. The term "random" refers to the changes that occurred in only one individual of the synthetic line. High similarities in frequencies for the total changes were observed between the two individual plants and in each of the different types. In RAPD and ISSR analyses, the numbers of consistent variant bands were 41 and 94, respectively, whereas the numbers of random bands were not more than 3. In methylation-sensitive RAPD analysis, the consistent variants were 22 , and the random variants were 0 and 1 . Likewise, in the corresponding ISSR analysis, the consistent variants were 38 and the random variants were 6 and 4. (Tables S2 and S3; Figure 4). Clearly, these data suggested that most genetic and methylation alterations might be non-random. Notably, the changes were often conserved in the CS, where at least $50 \%$ of the bands showing consistent changes (Figure 4) were identical to the variant bands of the CS. Some bands showing random genetic and methylation alterations were identical to the variant bands of the CS, implying that some random changes persist over evolutionary time. In the subsequent Southern blot validation, the detection of similar patterns between the two individual plants of synthetic wheat and between synthetic and natural wheat further corroborated the consistency of genomic changes, suggesting their non-random nature to some extent (Figure 5). 

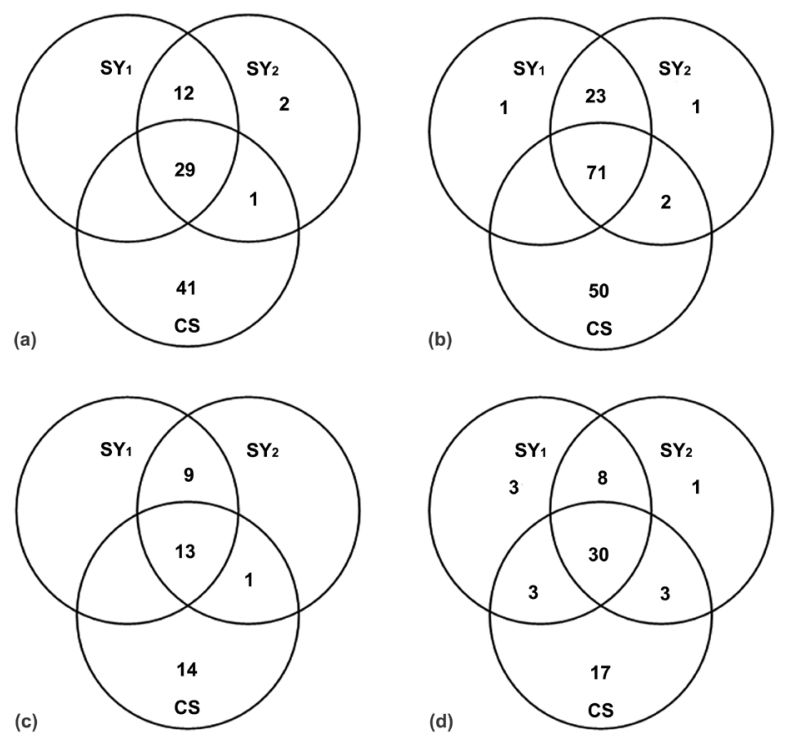

Figure 4. Diagram of the numbers of genomic variations shared by all three allohexaploid wheat lines: SY1, SY2 and CS, or two of them revealed by different markers. Total genetic changes identified in randomly amplified polymorphic DNA (RAPD) (a) and inter-simple sequence repeat (ISSR) (b). Total DNA methylation changes in methylation-sensitive RAPD (c) and ISSR (d).

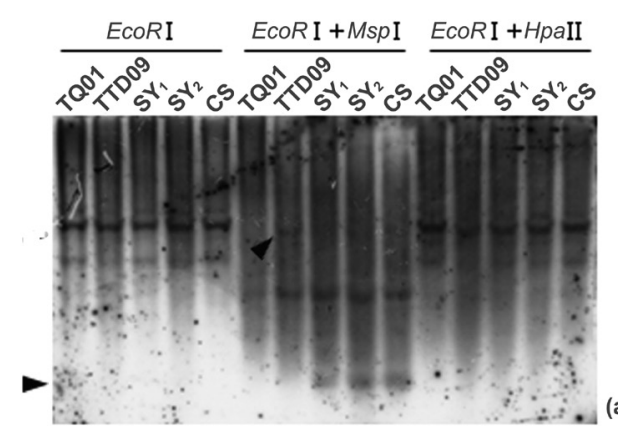

(a)

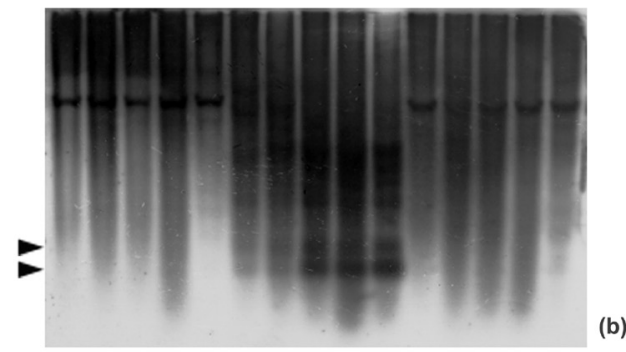

Figure 5. Validation of cytosine methylation alterations in the synthetic allohexaploid wheat as detected by Southern blotting probed by randomly amplified polymorphic DNA (RAPD) (a) and inter-simple sequence repeat (ISSR) (b) bands, respectively. The loss or gain of bands due to methylation alterations is denoted by arrows. The methylation-sensitive restriction enzymes (HpaII and MspI) and EcoRI used for digestion are labeled at the top.

Genetics and Molecular Research 16 (2): gmr16029303 


\section{Correlation of allohexaploidization-induced genetic and epigenetic changes in wheat}

By comparing the conventional and methylation-sensitive RAPD/ISSR analyses, we could detect correlations between the genetic and epigenetic changes. Some bands showing alterations in methylation were accompanied by genetic changes, while others were not, and the bands that did not appear in the amplification of the non-digested genome DNAs were not included. In these two cases, the former affected $2.42-3.91 \%$ of the bands observed in the synthetic allohexaploid individuals, while the latter influenced 4.66-5.45\% of the bands (including two markers, Table S3). Among the bands showing methylation changes, the percentages of the altered bands showing both methylation and genetic changes ranged from $18.18-25 \%$ for the synthetic wheat and $28.30-35.71 \%$ for the CS (including two markers). Among the bands showing genetic changes, the percentages of the bands showing both methylation and genetic changes ranged from 9.28 (e.g., 9 of 97)-11.58\% for the synthetic wheat and $12.20-14.08 \%$ for the CS (Table S3). Likewise, of the bands that showed methylation changes, $30.95-40.91 \%$ of the synthetic wheat bands and $25-28.30 \%$ of the CS bands showed no genetic variations. Unfortunately, the genetic changes without any methylation alterations could not be identified since they were ruled out as PCR artifacts, but there is no reason to deny their existence. Interestingly, among the two types of methylation changes-those without any genetic changes and those with genetic changes- the percentage of only the former out of the total methylation changes for the CS $(25 \%$ for RAPDs, $28.3 \%$ for ISSRs, Table S3) was lower than those of the two synthetic allohexaploid wheat individuals, suggesting that the methylation alterations independent of genetic variation were likely more stable over a longer evolutionary timescale.

In addition to exact correlations, cryptic correlations were detected between genetic and epigenetic alterations. We tested for possible correlations between various genetic and cytosine methylation-pattern changes using Pearson's correlation test (Table 1). The correlations between three genetic change factors, namely "loss of maternal bands", "novel bands" or "collective genetic changes", and "collective epigenetic changes", were all significant $(\mathrm{P}<$ $0.05)$. Correlations between various types of methylation changes accompanied by genetic changes and various genetic changes were significant $(\mathrm{P}<0.01$ or $\mathrm{P}<0.05)$, perhaps as a

Table 1. Bivariate correlation analysis of various types of genetic and epigenetic changes by SPSS

\begin{tabular}{l|c|c|c|c}
\hline & $\begin{array}{c}\text { Loss of maternal } \\
\text { parental bands }\end{array}$ & $\begin{array}{c}\text { Loss of paternal } \\
\text { parental bands }\end{array}$ & $\begin{array}{c}\text { Novel } \\
\text { Bands }\end{array}$ & $\begin{array}{c}\text { Collective } \\
\text { genetic changes }\end{array}$ \\
\hline CG Hypo & 0.075 & $-0.917^{* * \mathrm{~b}}$ & 0.184 & -0.069 \\
\hline CHG Hypo & 0.614 & $-0.852^{* \mathrm{c}}$ & 0.655 & 0.491 \\
\hline CG Hyper & 0.136 & 0.087 & 0.248 & 0.263 \\
\hline CHG Hyper & 0.689 & 0.402 & 0.543 & 0.740 \\
\hline Collective epigenetic changes & $0.898^{*}$ & -0.621 & $0.888^{*}$ & $0.818^{*}$ \\
\hline NGd-CG Hypo & 0.594 & 0.369 & 0.553 & -0.714 \\
\hline NG-CHG Hypo & -0.438 & -0.498 & -0.397 & - \\
\hline NG-CG Hyper & - & - & - & -0.575 \\
\hline NG-CHG Hyper & -0.713 & 0.334 & -0.804 & -0.770 \\
\hline Collective NG-epigenetic changes & -0.660 & 0.118 & -0.730 & -0.750 \\
\hline Ge-CG Hypo & $0.894^{*}$ & 0.098 & $0.813^{*}$ & $0.938^{* *}$ \\
\hline G-CHG Hypo & $0.964^{* *}$ & -0.358 & $0.932^{* *}$ & $0.948^{* *}$ \\
\hline G-CG Hyper & - & - & - & - \\
\hline G-CHG Hyper & $0.840^{*}$ & -0.164 & $0.815^{*}$ & $0.860^{*}$ \\
\hline Collective G-epigenetic changes & $0.969^{* *}$ & -0.220 & $0.928^{* *}$ & $0.977^{* *}$ \\
\hline
\end{tabular}

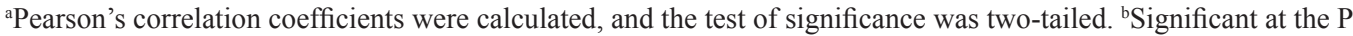

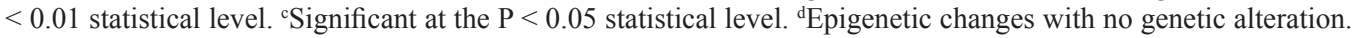

${ }^{\circ}$ Epigenetic changes with simultaneous genetic alteration. 
result of the genetic component contained by the former. As one of the more intriguing aspects of this analysis, the "loss of paternal bands" was the only genetic change factor significantly correlated with "CG hypomethylation change" $(\mathrm{P}<0.01)$ and "CHG hypomethylation change" $(\mathrm{P}<0.05)$.

\section{DNA sequencing and analysis of selected variant bands}

The BlastX or BlastN analysis of the 14 isolated and sequenced bands showing genetic or methylation variations revealed that these clones could be classified into three distinct classes. All sequences showed homology to the known-function genes and transposable element (TE)-related sequences (Table 2 and Table S4), with the exception of two sequences showing no database similarity.

Table 2. Functional classification of a subset of variant ISSR and RAPD bands in newly synthesized
allohexaploid wheat.
\begin{tabular}{l|c}
\hline Category & Numbers and percentages of variant ISSR and RAPD bands \\
\hline Known-function genes & $9(64.3 \%)$ \\
\hline Predicted genes & $1(7.1 \%)$ \\
\hline Transposable element (TE)-like sequences & $2(14.3 \%)$ \\
\hline No similarity & $2(14.3 \%)$ \\
\hline Total & $14(100 \%)$ \\
\hline
\end{tabular}

Detailed information regarding these variant bands is given in Table S4.

\section{Southern blot hybridization for validation}

Six DNA fragments showing the above-mentioned methylation changes were randomly selected to be used as probes in Southern blot hybridization. Methylation changes were clearly confirmed (Figure 5). Furthermore, the validated alterations occurred predominantly at CNG and not CG sites, supporting the notion that plant CNG methylation is more prone to disruption by stresses (Ou et al., 2009). 1) Almost no polymorphism was detected for IS17 between the parents, thus precluding further confirmation of the changes. 2) RA6 displayed a novel fragment in the EcoRI $+M s p I I-d i g e s t e d$ patterns but showed perfect additivity in the EcoRI alone- and EcoRI + HpaII-digested patterns, indicating that the external cytosine sites were demethylated. 3) For RA7, RA3, and IS11, the allohexaploid individuals yielded a pattern that was additive of both parents, but the intensity of bands in the EcoRI $+M s p I$-digested patterns of the synthetic and CS individuals appeared to be stronger than that of the corresponding bands in parents, suggesting that the external cytosine sites were partly demethylated. 4) We chose one clone to validate the result: RA2, which had two possibilities of alteration: methylation changes or sequence change at relevant restriction sites. RA2 exhibited deviation from additivity in the EcoRI + $M s p$ I-digested patterns, with a novel band appearing in the allohexaploid individuals and a loss of a parental band at a different position. These findings not only demonstrated the hypomethylation and hypermethylation of external cytosine sites, but also served as an example of the non-concordant variation in different CCGG sites.

Genetics and Molecular Research 16 (2): gmr16029303 


\section{DISCUSSION}

\section{Extensive genetic and epigenetic variations occurred in synthetic allotetraploid wheat}

Most previous studies that examined genomic changes associated with allopolyploidy in plants were based on a limited set of preselected loci using Southern blot analysis (Liu et al., 1998; Ozkan et al., 2001; Han et al., 2005). We previously reported rapid genomic changes associated with allohexaploid wheat formation from a genome-wide and unbiased perspective (Dong et al., 2005). However, our analysis was based on only one type of genetic marker, making the generality of the detected changes uncertain; we also did not analyze the correlations between genetic and epigenetic changes. Here, we applied RAPD and ISSR markers to simultaneously assess the allohexaploidy-associated genetic and epigenetic changes, providing basic and important evidence for investigating allohexaploid wheat.

In this study, we observed that extensive genetic and epigenetic changes occurred with the magnitudes of $30.7-39.5 \%$ and $13.33-15.66 \%$, respectively; these changes were broadly in agreement with previous reports (Shaked et al., 2001; Dong et al., 2005; Nie et al., 2008). Previous studies reported the changed frequencies of 20 and $48.6 \%$ via amplified fragment length polymorphism (AFLP) and restriction fragment length polymorphism (RFLP), respectively (Liu et al., 1998; Dong et al., 2005). Given the preponderance of genetic stability in other species and allohexaploid wheat lines, the phenomenon of extensive genomic alterations is indeed a species- and genotype-dependent trait in wheat. Notably, in the conventional analysis, the maternal parent that donated both its nucleus and cytoplasm experienced less loss than the paternal one that donated only its nucleus, a phenomenon that has also been reported in wheat and Brassica polyploidy (Qi et al., 2010). It seemed that the direction of genetic changes was influenced by the cytoplasmic-nuclear interactions, and thus supported the "nucleo-cytoplasmic interaction hypothesis". Meanwhile, the observed changes also supported another hypothesis stating that the parent with a higher ploidy level, i.e., the maternal parent (TTD09), would adapt to another episode of polyploidy shock since it had already undergone polyploidy (Song et al., 1995; Nie et al., 2008).

\section{Correlations between genetic and epigenetic alterations}

Extensive epigenetic alterations have been detected in almost every kind of plant polyploidization, and it is well known that epigenetics is involved in rebalancing the duplicated gene expression, genomic stability, and adaption (Jiao et al., 2015). However, it is difficult to elucidate the function of epigenetics in polyploidization because the epigenetic variations are always accompanied by genetic variations. Some epigenetic variations are completely dependent on their genetic component, and should be overlooked as the byproducts of genetic variations. However, we detected pure epigenetic variations independent of any genetic variations. Therefore, investigating their relationship is essential before we can elucidate the functions of epigenetic variations in polyploidization. The percentages of methylation and genetic changes simultaneously occurring in the same loci were $2.42-3.91 \%$ for the synthetic wheat, which occurred in at least $20 \%$ of the total methylation changes and in approximately $10 \%$ of total genetic changes, respectively. Therefore, it is possible that the two kinds of changes were interrelated rather than occurring independently. It should be kept in mind that the value of the percentages of the genetic and DNA methylation changes was underestimated,

Genetics and Molecular Research 16 (2): gmr16029303 
based on the limited power to detect the genetic and epigenetic variations of the two molecular markers. In addition, the overlapping distribution of epigenetic and genetic variations could be randomly produced. To investigate their relationship, we executed a statistical analysis to detect possible between genetic and epigenetic variations Significant negative correlations were observed between the "loss of paternal bands", "CG hypomethylation change", and "CHG hypomethylation change", suggesting that the cytoplasmic-nuclear interactions were partially correlated with hypomethylation or an unknown link between genetic instability and hypomethylation, as reported in some human cancer cells (Suzuki et al., 2006). With respect to the total methylation changes, at least $30-40 \%$ of the observed epigenetic component was possibly independent of the genetic changes.

\section{Underlying mechanisms of genomic changes}

The genetic changes found in this study could be attributed to several factors, such as heterozygosity, PCR artifacts, intergenomic recombination, alterations in DNA methylation, activation of TEs, or malfunction of a normally functional anti-mutational system. The parental lines were homozygous, and the synthetic allohexaploid has been maintained through selffertilization since its creation. Therefore, the deviation was not caused by the heterozygosity of parents. Furthermore, it cannot be attributed to PCR artifacts since they were ruled out. The cytological examination of the material showed that the loss of a chromosome(s) and gross structural aberration were not the causes of deviation. Previous studies have proposed that the intergenomic recombination was not the major force driving the changes observed in allohexaploid wheat (Liu et al., 1998). Considering the significant correlations between genetic and epigenetic changes, the methylation changes must contribute to at least some of the rapid genome reorganization, conceivably through inducing point mutations, affecting chromatin structure and DNA duplication, and other ways. Another possibility put forth by McClintock (1984) was that hybridization might result in a widespread activation of TEs because of the instability of the new genome. Allopolyploidization might also compromise the functions of anti-mutational systems, such as the mismatch repair system. Possible mechanisms for generating DNA methylation changes could include both allelic and ectopic homeologous interactions, genome defense functions, and genetic changes.

The sequencing results indicate that the known-function genes and TEs tend to change, suggesting that they could alter their expression levels and then produce phenotypic variations visible to natural selection; for example, TEs might trigger a preprogrammed response to genomic stress (McClintock, 1984). The genetic changes would also likely ensure the diploidlike meiotic behavior and reduce parental incompatibility. Therefore, these genomic variations would contribute to the speciation and evolutionary success of allohexaploid wheat.

\section{Conflicts of interests}

The authors declare no conflict of interest.

\section{ACKNOWLEDGMENTS}

Research supported by the National Science Foundation of China (\#31170208 and $31470335)$.

Genetics and Molecular Research 16 (2): gmr16029303 


\section{REFERENCES}

Ainouche ML and Jenczewski E (2010). Focus on polyploidy. New Phytol. 186: 1-4. http://dx.doi.org/10.1111/j.1469$\underline{8137.2010 .03215 . \mathrm{x}}$

Ammiraju JSS, Dholakia BB, Santra DK, Singh H, et al. (2001). Identification of inter simple sequence repeat (ISSR) markers associated with seed size in wheat. Theor. Appl. Genet. 102: 726-732. http://dx.doi.org/10.1007/ $\underline{\mathrm{s} 001220051703}$

Dong YZ, Liu ZL, Shan XH, Qiu T, et al. (2005). Allopolyploidy in wheat induces rapid and heritable alterations in DNA methylation patterns of cellular genes and mobile elements. Genetika 41: 1089-1095.

Feldman M (2001). Origin of cultivated wheat. In: The World Wheat Book (Bonjean AP and Angus WJ, eds.). Lavoisier Publishing, Paris, France, 3-56.

Feldman M, Levy AA, Fahima T and Korol A (2012). Genomic asymmetry in allopolyploid plants: wheat as a model. $J$. Exp. Bot. 63: 5045-5059. http://dx.doi.org/10.1093/jxb/ers192

Guo WL, Gong L, Ding ZF, Li YD, et al. (2006). Genomic instability in phenotypically normal regenerants of medicinal plant Codonopsis lanceolata Benth. et Hook. f., as revealed by ISSR and RAPD markers. Plant Cell Rep. 25: 896906. http://dx.doi.org/10.1007/s00299-006-0131-8

Han F, Fedak G, Guo W and Liu B (2005). Rapid and repeatable elimination of a parental genome-specific DNA repeat (pGc1R-1a) in newly synthesized wheat allopolyploids. Genetics 170: 1239-1245. http://dx.doi.org/10.1534/ genetics.104.039263

Hegarty MJ and Hiscock SJ (2008). Genomic clues to the evolutionary success of polyploid plants. Curr. Biol. 18: R435-R444. http://dx.doi.org/10.1016/j.cub.2008.03.043

Jiao J, Wu J, Lv Z, Sun C, et al. (2015). Methylation-sensitive amplified polymorphism-based genome-wide analysis of cytosine methylation profiles in Nicotiana tabacum cultivars. Genet. Mol. Res. 14: 15177-15187. http://dx.doi. org/10.4238/2015.November.25.6

Kidwell KK and Osborn TC (1992). Simple plant DNA isolation procedures. In: Plant Genomes: Methods for Genetic and Physical Mapping (Beckman JS and Osborn TC, eds.). Kluwer Academic Publishers, Dordrecht, The Netherlands, $1-13$.

Kjølner S, Såstad SM, Taberlet P and Brochmann C (2004). Amplified fragment length polymorphism versus random amplified polymorphic DNA markers: clonal diversity in Saxifraga cernua. Mol. Ecol. 13: 81-86. http://dx.doi. org/10.1046/j.1365-294X.2003.02037.x

Kraitshtein Z, Yaakov B, Khasdan V and Kashkush K (2010). Genetic and epigenetic dynamics of a retrotransposon after allopolyploidization of wheat. Genetics 186: 801-812. http://dx.doi.org/10.1534/genetics.110.120790

Li AL, Geng SF, Zhang LQ, Liu DC, et al. (2015). Making the bread: Insights from newly synthesized allohexaploid wheat. Mol. Plant 8: 847-859. http://dx.doi.org/10.1016/j.molp.2015.02.016

Liu B and Wendel JF (2002). Non-Mendelian phenomena in allopolyploid genome evolution. Curr. Genomics 3: 489-505. http://dx.doi.org/10.2174/1389202023350255

Liu B, Vega JM and Feldman M (1998). Rapid genomic changes in newly synthesized amphiploids of Triticum and Aegilops. II. Changes in low-copy coding DNA sequences. Genome 41: 535-542. http://dx.doi.org/10.1139/g98-052

Liu B, Xu C, Zhao N, Qi B, et al. (2009). Rapid genomic changes in polyploid wheat and related species: implications for genome evolution and genetic improvement. J. Genet. Genomics 36: 519-528. http://dx.doi.org/10.1016/S1673$\underline{8527(08) 60143-5}$

Madlung A and Wendel JF (2013). Genetic and epigenetic aspects of polyploid evolution in plants. Cytogenet. Genome Res. 140: 270-285. http://dx.doi.org/10.1159/000351430

McClelland M, Nelson M and Raschke E (1994). Effect of site-specific modification on restriction endonucleases and DNA modification methyltransferases. Nucleic Acids Res. 22: 3640-3659. http://dx.doi.org/10.1093/nar/22.17.3640

McClintock B (1984). The significance of responses of the genome to challenge. Science 226: 792-801. http://dx.doi. org/10.1126/science. 15739260

Nie LH, Han ZF, Lu LH, Yao YY, et al. (2008). Sequence variation study on genome and gene regions between synthetic hexaploid wheat and its parents. Prog. Nat. Sci. 18: 45-50. http://dx.doi.org/10.1016/j.pnsc.2007.09.006

Ou X, Long L, Zhang Y, Xue Y, et al. (2009). Spaceflight induces both transient and heritable alterations in DNA methylation and gene expression in rice (Oryza sativa L.). Mutat. Res. 662: 44-53. http://dx.doi.org/10.1016/j. mrfmmm.2008.12.004

Ozkan H, Levy AA and Feldman M (2001). Allopolyploidy-induced rapid genome evolution in the wheat (AegilopsTriticum) group. Plant Cell 13: 1735-1747. http://dx.doi.org/10.1105/tpc.13.8.1735

Qi B, Zhong X, Zhu B, Zhao N, et al. (2010). Generality and characteristics of genetic and epigenetic changes in

Genetics and Molecular Research 16 (2): gmr16029303 
newly synthesized allotetraploid wheat lines. J. Genet. Genomics 37: 737-748. http://dx.doi.org/10.1016/S1673$\underline{8527(09) 60091-6}$

Rapp RA and Wendel JF (2005). Epigenetics and plant evolution. New Phytol. 168: 81-91. http://dx.doi.org/10.1111/ j.1469-8137.2005.01491.x

Shaked H, Kashkush K, Ozkan H, Feldman M, et al. (2001). Sequence elimination and cytosine methylation are rapid and reproducible responses of the genome to wide hybridization and allopolyploidy in wheat. Plant Cell 13: 1749-1759. http://dx.doi.org/10.1105/tpc.13.8.1749

Song K, Lu P, Tang K and Osborn TC (1995). Rapid genome change in synthetic polyploids of Brassica and its implications for polyploid evolution. Proc. Natl. Acad. Sci. USA 92: 7719-7723. http://dx.doi.org/10.1073/pnas.92.17.7719

Suzuki K, Suzuki I, Leodolter A, Alonso S, et al. (2006). Global DNA demethylation in gastrointestinal cancer is age dependent and precedes genomic damage. Cancer Cell 9: 199-207. http://dx.doi.org/10.1016/j.ccr.2006.02.016

WendelJF(2000).Genomeevolutioninpolyploids.PlantMol.Biol.42:225-249.http://dx.doi.org/10.1023/A:1006392424384

Wolfe AD, Xiang Q-Y and Kephart SR (1998). Assessing hybridization in natural populations of Penstemon (Scrophulariaceae) using hypervariable intersimple sequence repeat (ISSR) bands. Mol. Ecol. 7: 1107-1125. http:// dx.doi.org/10.1046/j.1365-294x.1998.00425.x

Zhao N, Xu L, Zhu B, Li M, et al. (2011a). Chromosomal and genome-wide molecular changes associated with initial stages of allohexaploidization in wheat can be transit and incidental. Genome 54: 692-699. http://dx.doi.org/10.1139/ g11-028

Zhao N, Zhu B, Li M, Wang L, et al. (2011b). Extensive and heritable epigenetic remodeling and genetic stability accompany allohexaploidization of wheat. Genetics 188: 499-510. http://dx.doi.org/10.1534/genetics.111.127688

\section{Supplementary material}

Table S1. RAPD and ISSR primers used in this study.

Table S2. Genetic changes in the individuals of synthetic allohexaploid wheat (designated SY1 and SY2) and their natural counterpart (Common wheat cultivar, Chinese Spring, CS) in the conventional RAPD and ISSR analysis, as revealed by the numbers and percentages of bands from parents and allohexaploid wheat.

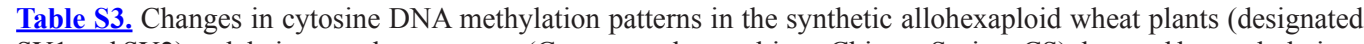
SY1 and SY2) and their natural counterpart (Common wheat cultivar, Chinese Spring, CS) detected by methylationsensitive RAPD and ISSR.

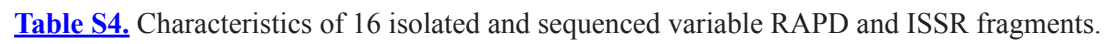

